needs; lack of communication between multiple providers; distress at having providers release children to full activities before caregivers were comfortable. At 6 months, approximately 24\% of parents reported children had ongoing cognitive limitations, 29\% reported emotional problems, $19 \%$ reported physical limitations, $33.3 \%$ reported difficulty in school, and 15\% reported play/social difficulties. DISCUSSION/SIGNIFICANCE OF IMPACT: Evidence suggests families face significant barriers in accessing follow-up care, despite nearly universal health insurance coverage for children. Further, a large percentage of parents report ongoing health needs, despite the majority of the cohort having only mild or moderate severity injuries. Making follow-up care more patient-centered for families of traumatically injured children may improve compliance with medical regiments and reduce the likelihood of future disability. Examples of this may be coordinating care among multiple specialty providers, so that patients with multiple injuries can schedule multiple follow-up appointments on the same day. Additionally, more caregiver education on administering pain medication, caring for wounds, and safe practices for returning to full activities would be beneficial for families.

\section{Cell Proliferation and Differentiation in 3D printed Polycarbonate Urethane Porous Scaffolds}

Bijan Abar ${ }^{1}$, Alejandro Aalleja, Cambre Kelly, Natalia Von Windheim, Jennifer West and Kenneth Gall

${ }^{1}$ Duke University

OBJECTIVES/SPECIFIC AIMS: The aim of this study is to understand how porosity and collagen filling impact cell proliferation and differentiation in 3D printed scaffolds. METHODS/STUDY POPULATION: 3 groups of scaffolds will be 3D printed using FDM: solid scaffold, porous scaffold and porous scaffold with collagen gel ( $\mathrm{n}=10$ for each group) Internal geometries and surface structure will be analyzed using micro CT and Scanning Electron Mi RESULTS/ ANTICIPATED RESULTS: We hypothesize that porosity and collagen filler will increase signal from Picogreen assay and ALP assay when normalized to scaffold surface area, indicating enhanced cell proliferation and differentiation. DISCUSSION/SIGNIFICANCE OF IMPACT: 3D printing PCU is a relatively new technique with very little published in the literature. Previous work has focused on the mechanical properties and not the biological response to the polymer. Understanding how to optimize cellular proliferation and differentiation can lead to the development of better implants that will integrate into the host's structure and facilitate tissue regeneration.

\section{3}

\section{Comparative Effectiveness of Levetiracetam versus Phenobarbital for Infants Aged One Month to One Year, using US Medicaid Claims \\ Michelle Yun ${ }^{1}$, Manyao Zhang ${ }^{1}$, Alan Wu ${ }^{1}$, Natasha Basma ${ }^{1}$ and Zachary Grinspan ${ }^{1}$ \\ ${ }^{1}$ Weill Cornell}

OBJECTIVES/SPECIFIC AIMS: Using national Medicaid claims, this retrospective cohort study aims to compare the outcomes of levetiracetam (LEV) versus phenobarbital (PHB) as initial monotherapy in infants with epilepsy aged one month to one year. We primarily analyzed health services outcomes, as follows: (1) Emergency Department (ED) visits: proportion of those with at least $1 \mathrm{ED}$ visit, time to first $\mathrm{ED}$ visit, total number of $\mathrm{ED}$ visits, proportion leading to inpatient admission (2) Inpatient Admissions: proportion of those with at least 1 admission, time to first admission, total number of inpatient admissions, total length of stay (3) Treatment discontinuation: proportion of those who discontinued medication, time to discontinuation, proportion of those with prescription of second antiepileptic drug (AED) (4) Cost: total cost at 1 year from first prescription. METHODS/STUDY POPULATION: The project is a retrospective cohort study utilizing Medicaid claims from 2009-2012 from all states and DC. Inclusion criteria were: (1) Epilepsy diagnosis (ICD9 345.x) at age 1 month to 1 year (2) 1 year of continuous Medicaid enrollment Exclusion criteria were: (1) Neither LEV nor PHB monotherapy as initial treatment (2) Neither LEV nor PHB prescription within 45 days of epilepsy diagnosis (3) Less than 1 year of follow-up after first LEV or PHB prescription (4) Infantile Spasms diagnosis (5) Brain Surgery (6) Death within 1 year of follow-up Using $\mathrm{R}$ for statistical analysis, we analyzed outcomes including ED visits, inpatient admissions, treatment discontinuation, and total cost. In this abstract, we present our preliminary bivariate analysis. RESULTS/ANTICIPATED RESULTS: Demographics and Etiology: Compared to infants prescribed PHB $(n=1954)$, infants prescribed LEV $(\mathrm{n}=1248)$ were older (median 6 months [IQR 3-9] vs 3[2-6]; $\mathrm{p}<0.001)$, more likely to be white $(44.2 \%$ vs $38.9 \%$; $\mathrm{p}<0.05)$ and not Hispanic $(63.5 \%$ vs $58.9 \%$; $\mathrm{p}<0.05)$. There were also important differences in epilepsy etiologies $(\mathrm{p}<0.05)$. For example, infants prescribed LEV were more likely to have a diagnosis of tuberous sclerosis ( $1 \%$ vs $0.15 \%$ ) or traumatic brain injury ( $12.8 \%$ vs $0.56 \%)$. Health Services Outcomes: After 1 year, infants prescribed LEV had more ED visits $(2[0-4]$ vs $1[0-3] ; p<0.001)$ but shorter inpatient length of stay when admitted (3 days [2-5] vs 3 [2-6]; $\mathrm{p}<0.001)$. They were less likely to discontinue the medication $(46.6 \%$ vs $64.3 \% ; \mathrm{p}<0.001)$ but more likely to have a second AED prescription $(53.3 \%$ vs $43.4 \%$; $p<0.001)$. Other outcomes, including total cost, were similar. DISCUSSION/SIGNIFICANCE OF IMPACT: This preliminary analysis suggests that the healthcare trajectory of infants treated with LEV and PHB differ in complex ways. In ongoing work, we are conducting a multivariable comparative effectiveness analysis of LEV versus $\mathrm{PHB}$ using propensity score weighting to account for observable selection bias and multiple imputation to account for missing data.

3245

Comparison of Health Outcomes in Adolescent and Young Adult (AYA) Oncology Patients Treated at a Pediatric versus Adult Institute

Amy Moskop ${ }^{1}$, Julie Panepinto and Sadie Dobrozsi

${ }^{1}$ Medical College of Wisconsin

OBJECTIVES/SPECIFIC AIMS: Objectives: To examine the differences in health outcomes of adolescents and young adults treated for malignancy at a pediatric cancer center compared to an adult cancer center. To determine the differences in receipt of supportive care for adolescents and young adults with malignancy who are treated at a pediatric cancer center compared to an adult cancer center. METHODS/STUDY POPULATION: Methods: This will be a retrospective cohort study of patients from the ages of 15 to 